\title{
Cosmological constant, brane tension and large hierarchy in a generalized Randall-Sundrum braneworld scenario
}

\author{
Saurya Das $^{1 *}$, Debaprasad Maity ${ }^{2} \dagger$ and Soumitra SenGupta ${ }^{3} \ddagger$ \\ ${ }^{1}$ Department of Physics, University of Lethbridge \\ 4401 University Drive, Lethbridge, \\ Alberta - T1K 3M4, Canada \\ ${ }^{2}$ The Institute of Mathematical Sciences, \\ CIT Campus, Chennai - 600 113, India \\ ${ }^{3}$ Department of Theoretical Physics and Centre for Theoretical Sciences, \\ Indian Association for the Cultivation of Science, \\ Kolkata - 700 032, India
}

\begin{abstract}
We consider a generalized Randall Sundrum (RS) brane world scenario with a cosmological constant $\Omega$ induced on the visible brane. We show that for $\Omega<0$, resolution of the hierarchy problem requires an upper bound on the magnitude of $\Omega$. The corresponding tension on the visible brane can be positive or negative. On the other hand, there is no such bound for $\Omega>0$. However, in this case, the resolution of the hierarchy problem along with the tuning of the value of the cosmological constant to its observed value closed to $+10^{-124}$ (in Planck units) naturally lead to the tuning of the modulus to a small value of inverse Planck length as estimated in the original RS scenario.
\end{abstract}

Some of the intriguing questions about our physical universe, which remain unanswered, are:

(i) Why does it appear to have $(3+1)$ space-time dimensions? Are there additional unobserved dimensions?

(ii) Why is the ratio of the electroweak scale/Higgs mass $(m)$ to the Planck mass $\left(m_{0}\right)$ so tiny $\left(\simeq 10^{-16}\right)$ ? This gives rise to the gauge hierarchy problem.

(iii) Why is the observed value of the cosmological constant $\Omega$ extremely small $\left(\Omega \simeq 10^{-124}\right)$ (in Planck units)? This gives rise to the cosmological fine tuning problem.

In the so-called brane world models proposed during the last decade [1, 2, 3] , it was shown that questions (i) and (ii) may be related, in the sense that if one assumes that the space-time dimension exceeds four, the hierarchy problem can be solved. A lot of work has also been done recently in an attempt to relate the questions (i) and (iii) by adopting various approaches such as the domain wall scenarios [4, 5] and self tuning mechanism in large extra dimensions [6, 7, 8]. While the RS two-brane model is particularly successful in resolving the fine tuning problem without bringing in any arbitrary intermediate scale between the Planck and the Tev scale, it has a somewhat unsatisfactory but inevitable feature of having a negative tension visible brane to describe our Universe. It has been shown that such negative tension branes are intrinsically unstable. Furthermore the effective visible 3 -brane being flat has zero cosmological constant which is not consistent with its presently observed small value. In this article we extend such warped geometric model to include a non-zero cosmological constant and look for a possible positive tension Tev brane when a large hierarchy exists between the two branes. A motivation to look for positive tension branes lies in string inspired brane

\footnotetext{
*E-mail: saurya.das@uleth.ca

${ }^{\dagger}$ E-mail: debu@imsc.res.in

${ }^{\ddagger}$ E-mail: tpssg@iacs.res.in
}

world scenarios, in which the relevant D-branes have positive tension.

Here by generalizing the RS model to include a nonvanishing cosmological constant on the visible brane, we show that questions (i), (ii) and (iii), as well as the issue of brane tensions are intimately related. We demonstrate that while the regime of positive cosmological constant on the visible 3-brane (de-Sitter) strictly implies a negative brane tension, that with negative cosmological constant (anti de-Sitter) admits of both positive and negative tensions of the visible brane. For both the regions, the desired warping from Planck to Tev scale can be achieved as a proper resolution of the gauge hierarchy problem. However larger is the magnitude of the $4 \mathrm{~d}$ cosmological constant $(+$ ve or - ve) further away is the value of the modulus from the Planck length leading to a new hierarchy of scales. This obviously brings back the fine tuning problem in a new guise and is undesirable.

In the RS scenario, it was proposed that our universe is five dimensional, described by the metric [1]:

$$
d s^{2}=e^{-2 k r y} \eta_{\mu \nu} d x^{\mu} d x^{\nu}+r^{2} d y^{2}
$$

where Greek indices $\mu, \nu, \ldots$ run over $0,1,2,3$ and refer to the 4 observed dimensions, $y$ signifies the coordinate on the additional spacelike dimension of length $r, \Lambda$ is the bulk cosmological constant, $k \equiv \sqrt{-\Lambda / 12 M^{3}} \approx$ Planck mass. The factor $e^{-2 k r y}$ is known as the warp factor. The geometry of the extra dimension is orbifolded by $S^{1} / Z_{2}$. The constant $y$ slices at $y=0$ and $y=r \pi$ are known as the hidden and visible branes, the observable universe being identified with the latter which has a negative brane tension as opposed to the hidden brane which has a positive brane tension. It can be shown that even if the Higgs (or any other) mass parameter in the fivedimensional Lagrangian is of the order of Planck scale $m_{0}\left(\approx 10^{16} \mathrm{TeV}\right)$, on the visible four dimensional brane, it gets 'warped' by a factor of the form:

$$
m=m_{0} e^{-2 k r \pi} \text {. }
$$


For, $k r \simeq 11.84$, one gets $m \approx 1 \mathrm{TeV}$. Thus, in this picture, the origin of a small Higgs mass lies in the warped geometry of five dimensional spacetime.

In [1], it was also shown that the cosmological constant induced on the visible brane is zero. In this paper we demonstrate that the last condition can be relaxed for a more general warp factor, such that the metric is given by:

$$
d s^{2}=e^{-2 A(y)} g_{\mu \nu} d x^{\mu} d x^{\nu}+r^{2} d y^{2} .
$$

For the above metric the visible brane can have a negative or a positive cosmological constant. Defining $e^{-A(k r \pi)}=$ $m / m_{0}=10^{-n}$ and the magnitude of the induced cosmological constant $=10^{-N}$ (in Planck units), we show that for negative cosmological constant $N$ cannot be less than a minimum value given by $N_{\min }=2 n$. This implies a very small upper bound of the magnitude of the cosmological constant. Although for positive value of $\Omega$ no such bound exists in general, the need for resolution of the hierarchy problem without introducing a new scale in the theory (i.e keeping the value of the modulus close to Planck length ), restricts the cosmological constant to be very very small. The corresponding brane tension for both Tev and the Planck branes are determined for these two different scenarios.

We start with the metric (3) and evaluate the function $A(y)$ which extremises the action:

$$
S=\int d^{5} x \sqrt{-G}\left(M^{3} \mathcal{R}-\Lambda\right)+\int d^{4} x \sqrt{-g_{i}} \mathcal{V}_{i}
$$

where $\Lambda$ is the bulk cosmological constant, $\mathcal{R}$ is the bulk (5-dimensional) Ricci scalar and $\mathcal{V}_{i}$ is the tension of the $i^{\text {th }}$ brane ( $i=h i d(v i s)$ for the hidden (visible) brane). Note that $g_{\mu \nu}$ is the four dimensional metric.

The resulting Einstein equations are:

$$
\begin{aligned}
{ }^{4} G_{\mu \nu}-g_{\mu \nu} e^{-2 A}\left[-6 A^{\prime 2}+3 A^{\prime \prime}\right] & =-\frac{\Lambda}{2 M^{3}} g_{\mu \nu} e^{-2 A}(5) \\
-\frac{1}{2} e^{2 A}{ }^{4} R+6 A^{\prime 2} & =-\frac{\Lambda}{2 M^{3}}
\end{aligned}
$$

with the boundary conditions

$$
\left[A^{\prime}(y)\right]_{i}=\frac{\epsilon_{i}}{12 M^{3}} \mathcal{V}_{i}
$$

where $\epsilon_{\text {hid }}=-\epsilon_{v i s}=1$. In the above, ${ }^{4} G_{\mu \nu}$ and ${ }^{4} R$ are the four dimensional Einstein tensor and Ricci scalar respectively, defined with respect to $g_{\mu \nu}$. Dividing both sides of Eq.(5) by $g_{\mu \nu}$, for any $\mu, \nu$, and rearranging terms, it is seen that one side contains $A(y)$ and its derivatives, depending on the extra coordinate $y$ alone, while the other side depends on the brane coordinates $x^{\mu}$ alone [5, 9]. Thus each side is equal to an arbitrary constant, $\Omega$ say. Thus, we get from Eq.(6) [11] :

$$
\begin{gathered}
{ }^{4} G_{\mu \nu}=-\Omega g_{\mu \nu} \\
e^{-2 A}\left[-6 A^{\prime 2}+3 A^{\prime \prime}-\frac{\Lambda}{2 M^{3}}\right]=-\Omega .
\end{gathered}
$$

Computing ${ }^{4} R$ from Eq.(8), and substituting in Eq.(6), $A^{\prime}$ can be evaluated, which on further substitution in Eq.(9), yields a simplified expression for $A^{\prime \prime}$ :

$$
\begin{aligned}
6 A^{\prime 2} & =-\frac{\Lambda}{2 M^{3}}+2 \Omega e^{2 A} \\
3 A^{\prime \prime} & =\Omega e^{2 A} .
\end{aligned}
$$

The above corresponds to a constant curvature brane spacetime, as opposed to a Ricci flat spacetime, which is normally assumed. For example, for $\Omega>0$ and $\Omega<0, g_{\mu \nu}$ may correspond to dS-Schwarzschild and AdS-Schwarzschild spacetimes respectively.

For Ads bulk i.e. $\Lambda<0$, we first consider the regime for which the induced cosmological constant $\Omega$ on the visible brane is negative. Defining the parameter $\omega^{2} \equiv$ $-\Omega / 3 k^{2} \geq 0$, we get the following solution for the warp factor, satisfying Eqs.(10,11) :

$$
e^{-A}=\omega \cosh \left(\ln \frac{\omega}{c}+k y\right)
$$

Note that the RS solution $A=k y$ is recovered in the limit $\omega \rightarrow 0$. ¿From this and Eq.(17), the brane tensions follow:

$\mathcal{V}_{v i s}=12 M^{3} k\left[\frac{\frac{\omega^{2}}{c_{1}^{2}} e^{2 k r \pi}-1}{\frac{\omega^{2}}{c_{1}^{2}} e^{2 k r \pi}+1}\right] ; \mathcal{V}_{h i d}=12 M^{3} k\left[\frac{1-\frac{\omega^{2}}{c_{1}^{2}}}{1+\frac{\omega^{2}}{c_{1}^{2}}}\right]$

Normalizing the warp factor to unity at the orbifold fixed point $y=0$, we get:

$$
c_{1}=1+\sqrt{1-\omega^{2}} .
$$

(The other solution $c_{1}=1-\sqrt{1-\omega^{2}}$, for which the RS result is not recovered in the $\omega^{2} \rightarrow 0$ limit, is excluded from further discussions).

Next, to solve the hierarchy problem, we equate the warp factor at $y=r \pi$ to the ratio of the Higgs to the Planck mass:

$$
e^{-A}=\omega \cosh \left(\ln \frac{\omega}{c_{1}}+k r \pi\right)=10^{-n} .
$$

At this point, we keep $n$ arbitrary, although eventually we will assume it to be $\simeq 16$. Defining $k r \pi \equiv x$, the above equation simplifies to:

$$
10^{-n}=\frac{1}{2}\left[c_{1} e^{-x}+\frac{\omega^{2}}{c_{1}} e^{x}\right]
$$

from which one gets:

$$
e^{-x}=\frac{10^{-n}}{2}\left[1 \pm \sqrt{1-\omega^{2} 10^{2 n}}\right] .
$$

Clearly, real solutions for $e^{-x}$ exists if and only if $\omega^{2} \leq$ $10^{-2 n}$. In other words, solution of the hierarchy problem requires the magnitude of the induced cosmological constant on the brane to be extremely small! Thus from 
Eq.(14), $c_{1} \simeq 2$, which we will assume from now on. Further setting the brane cosmological constant $\omega^{2} \equiv 10^{-N}$, we get the upper bound on the cosmological constant:

$$
N_{\text {min }}=2 n \text {. }
$$

Thus, for $n=16$, it follows that the brane cosmological constant cannot exceed $10^{-32}$ (in Planck units).

Also Eqs.16) and (17) simplify to:

$$
\begin{aligned}
10^{-N} & =4\left(10^{-n} e^{-x}-e^{-2 x}\right), \\
e^{-x} & =\frac{10^{-n}}{2}\left[1 \pm \sqrt{1-10^{-(N-2 n)}}\right] .
\end{aligned}
$$

From Eq. 201 above, it can be seen that for $N \rightarrow \infty$ $\left(\omega^{2} \rightarrow 0\right)$, the RS value of $x=n \ln 10$ is recovered (the other solution in this limit, $x=\infty$, is excluded). For $N=N_{\text {min }}$, we get a degenerate solution $x=n \ln 10+\ln 2$. However, for $N<N_{\min }$, there are two values of $x$ which give rise to the required warping, instead of one, as was in the case of RS [12]. For $N-2 n \gg 1$, these two solutions are:

$x_{1} \simeq n \ln 10+\frac{1}{4} 10^{-(N-2 n)}, \quad x_{2} \simeq(N-n) \ln 10+\ln 4$.

The first corresponds to the RS value plus a minute correction, while the second, although of a similar order of magnitude (thus ensuring that no new scale is introduced), is quite distinct. The hierarchy problem is solved for two small and negative values of the cosmological constant. Note that $x_{2}>x_{1}$.

In Figs.(11) and (2), we have plotted $N$ versus $x$, using Eq.(19), we have plotted $-N$ versus $x$ (for $n=16$ ). In Fig.(1), point $\mathbf{A}$ corresponds to the RS values of $(x, N)=$ $(n \ln 10, \infty)$. Point $\mathbf{B}$ corresponds to the maximum value of $\omega^{2}$, i.e. $(x, N)=(n \ln 10+\ln 2,2 n)$, beyond which $\omega^{2}$ starts to decrease once again. Far from the maximum, $N$ is given approximately by the linear relation:

$$
N=(\ln 10)^{-1}[-x-n-\ln 4],
$$

which we have plotted in Fig.(21).

From Eqs. 131) and (20), we obtain the tension on the visible brane for the two solutions as:

$$
\mathcal{V}_{v i s}=\left(12 M^{3} k\right) \frac{1-10^{N-2 n}\left[1 \pm \sqrt{1-10^{-(N-2 n)}}\right]}{1+10^{N-2 n}\left[1 \pm \sqrt{1-10^{-(N-2 n)}}\right]}
$$

Observe that $\mathcal{V}_{\text {vis }}=0$ when $N=N_{\text {min }}=2 n$. Further, it is easy to show that $\mathcal{V}_{\text {vis }}<0$ for $x=x_{1}$, while $\mathcal{V}_{\text {vis }}>0$ for $x=x_{2}$. Thus the second solution for $x$ is associated with a positive tension brane, which also produces the desired large hierarchy. When $N-2 n \gg 1$, the two tensions are approximately given as:

$$
\begin{aligned}
& \mathcal{V}_{v i s-1} \simeq-\left(12 M^{3} k\right) \\
& \mathcal{V}_{v i s-2} \simeq \frac{1}{3}\left(12 M^{3} k\right) .
\end{aligned}
$$

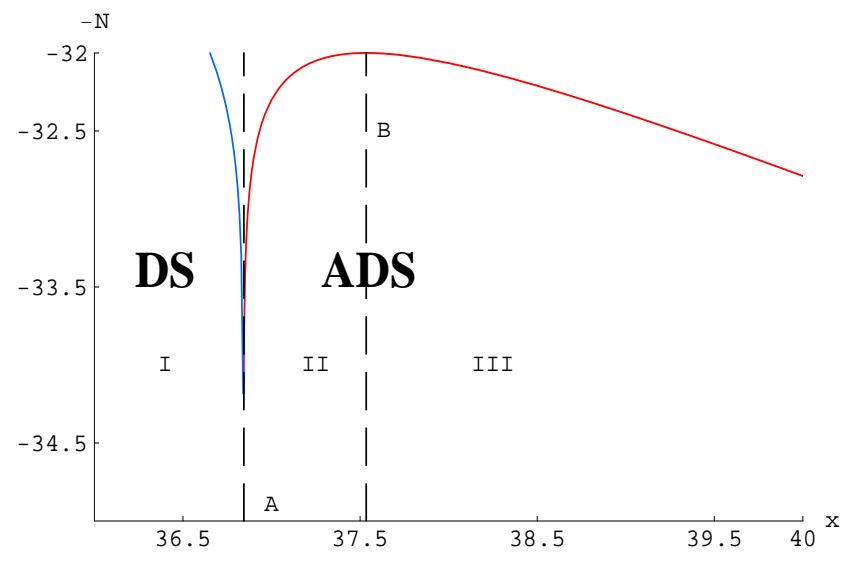

FIG. 1: Graph of $N$ versus $x=36-40$, for $n=16$ and for both positive and negative brane cosmological constant. The curve in region-I corresponds to positive cosmological constant on the brane, whereas the curve in regions-II \& III represents negative cosmological constant on the brane.

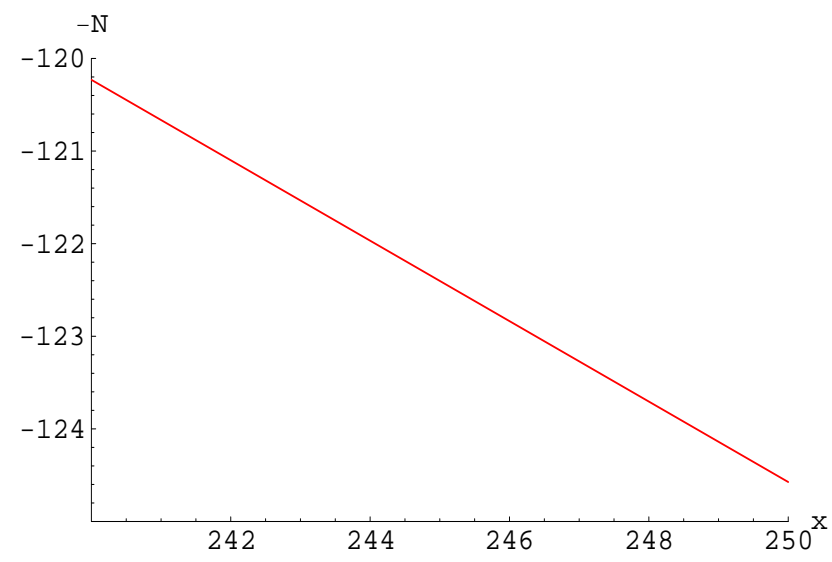

FIG. 2: (Continuation of of graph in Fig. 1) Graph of $N$ versus $x=240-250$, for $n=16$ and negative brane cosmological constant

¿From (25), we see that a small negative cosmological constant suffices to render the tension positive, provided the distance between the branes is somewhat larger than the value predicted by RS. From (13), the tension on the hidden brane on the other hand, is given by:

$$
\mathcal{V}_{\text {hid }}=\left(12 M^{3} k\right) \frac{4-10^{-N}}{4+10^{-N}},
$$

which is always positive.

Next, for $\Omega>0$, the warp factor which satisfies Eqs. (10) 111 is given by:

$$
e^{-A}=\omega \sinh \left(\ln \frac{c_{2}}{\omega}-k y\right),
$$

where now $\omega^{2} \equiv \Omega / 3 k^{2}$, and as before, normalization of the warp factor on the hidden brane gives $c_{2}=$ $1+\sqrt{1+\omega^{2}}$.

Equating the above to $m / m_{0}=10^{-n}$, we get:

$$
10^{-n}=\frac{1}{2}\left[c_{2} e^{-x}-\frac{\omega^{2}}{c_{2}} e^{x}\right]
$$


and the counterpart of Eq.(17) is now,

$$
e^{-x}=\frac{10^{-n}}{c_{2}}\left[1+\sqrt{1+\omega^{2} 10^{2 n}}\right] .
$$

From Eq.(29) one can see that in this case, there are no bounds on $\omega^{2}$, and the (positive) cosmological constant can be of arbitrary magnitude. Also, there is a single solution of $x$, whose value depends on $\omega^{2}$ and $n$. This is described in the region I in FIG.1, from where it can be seen that a small and positive value of the cosmological constant, say the observed value $\sim 10^{-124}$ (in Planckian units), corresponds to $x$ and hence $k r \pi$ very close to the RS value 36.84 and the value of the cosmological constant rises sharply with small departure from the RS value of $k r \pi$. This explains why the observed small value of cosmological constant naturally leads to the tuning of the value of the modulus $r$ to be inverse of Planck length when the value of $k$ is of the order of Planck mass. However in this regime, the Tev brane tension continues to be negative as in the RS case. This can be seen from the expressions for the brane tensions which in this case are:

$\mathcal{V}_{v i s}=12 M^{3} k\left[\frac{\frac{\omega^{2}}{c_{2}^{2}} e^{2 k r \pi}+1}{\frac{\omega^{2}}{c_{2}^{2}} e^{2 k r \pi}-1}\right] ; \mathcal{V}_{h i d}=12 M^{3} k\left[\frac{1+\frac{\omega^{2}}{c_{2}^{2}}}{1-\frac{\omega^{2}}{c_{2}^{2}}}\right]$

As $c_{2}>\omega, \mathcal{V}_{h i d}$ is always positive. On the other hand from Eq.(30), the condition of positivity of the warp factor $10^{-n}$ requires $\frac{\omega^{2}}{c_{2}^{2}} e^{2 k r \pi}<1$. This implies $\mathcal{V}_{v i s}$ is negative for the entire range of positive values of $\Omega$.

In summary, we have derived the exact form for the warp factor in a generalized RS braneworld scenario, which admits of both positive or negative cosmological constant on the visible 3-brane. We have shown that the induced negative cosmological constant on the 3-brane is bounded from below by $\sim-10^{-32}$ (in Planck units). Furthermore for a tiny value of cosmological constant, the hierarchy problem can be resolved for two different values of the modulus, one of which corresponds to a positive tension Tev brane along with the positive tension Planck brane. It would be interesting to study implications of these results. In the other regime namely $\Omega>0$ the Tev brane tension turns out necessarily to be negative. The value of the modulus corresponding to the observed value of the cosmological constant lies very close to the RS value and rises very rapidly as we depart from the value of $k r$ predicted in the original RS model. Tuning of the small cosmological constant thus implies the tuning of the value of the modulus $r$ at the inverse of Planck length. It may be noted that a modulus value much away from Planck length ( corresponding to a large cosmological constant ) will give rise to a new hierarchy of scales leading to a possible large radiative correction to the modulus and in turn bringing back the fine tuning problem again. Our results thus indicate that if one wants to resolve the fine tuning problem in connection with the Higgs mass, without bringing in any hierarchy through the size of the modulus, the value of the cosmological constant $\Omega$ (whether positive or negative) on the Tev brane must be very small! In other words the resolution of the gauge hierarchy problem, and the cosmological fine tuning problem are related and one implies the other if the modulus value is kept close to Planck length to avoid any further scale hierarchy. It will now be interesting to study whether for this generalised RS model the modulus can be stabilized to a value close to Planck length following the mechanism proposed by Goldberger and Wise [10] and what are the other phenomenologi$\mathrm{cal} /$ cosmological implications of such a generalised warp factor and brane tensions. We hope to report these in future works.

We thank A. Dasgupta, A. Sen and S. Sur for useful discussions. SD thanks the Department of Theoretical Physics, Indian Association for the Cultivation of Science, Kolkata, for hospitality, where this work was done. This work is supported by the Natural Sciences and Engineering Research Council of Canada.
[1] L. Randall and R. Sundrum, Phys. Rev. Lett. 833370 (1999); ibid Phys. Rev. Lett. 834690 (1999).

[2] N. Arkani-Hamed, S. Dimopoulos and G. Dvali, Phys. Lett. B429 263 (1998); I. Antoniadis, N. Arkani-Hamed, S. Dimopoulos and G. Dvali, Phys. Lett. B436 257 (1998).K.Akama, Prog. Theor. Phys. 80, 935 (1988); T.Delsate et.al. gr-qc/0605039 hep-th/0602172.

[3] A. G. Cohen and D. B. Kaplan, Phys. Lett. B470 , 52 (1999); I. Antoniadis, S. Dimop oulos and A. Giveon, JHEP, 05, 055 (2001); T. Multamaki and I. Vilja, Phys. Lett. B545, 389 (2002); C. P. Burgess, J. M. Cline, N. R. Constable and H. Firouzjahi, JHEP, 01, 014 (2002).

[4] V.A. Rubakov and M.E. Shaposhnikov, Phys. Lett. B125, 136 (1983); Phys. Lett. B125, 139 (1983).

[5] E. and H. Verlinde, JHEP 0005, 034 (2000), hep-th/9912018;C. Schmidhuber, Nucl. Phys. B 580, 140 (2000), [e-Print: hep-th/9912156],

[6] S. P. de Alwis, Nucl. Phys. B597, 263 (2001); A. Krause,
JHEP, 09, 016 (2003); S. Kachru, M. B. Schulz, E. Silverstein, Phys. Rev. D62, 045021 (2000), N. A. Hamed et al, Phys. Lett. B 480, 193 (2000).

[7] I. Navarro, Class. Quant. Grav. 20, 3603 (2003); I. Navarro, JCAP, 09, 004 (2003); S. M. Carroll and M. M. Guica, hep-th/0302067; J. M. Cline, J. Descheneau, M. Giovannini and J. Vinet, JHEP, 06, 048 (2003); Y. Aghababaie, C. P. Burgess, S. L. Parameswaran and F. Quevedo, Nucl. Phys. B680, 389 (2004); E. K. Park, P. S. Kwon, hep-th/0702171, referances therein.

[8] C. Csaki et al Nucl. Phys. B584, 359 (2000); H. P. Nilles, A. Papazoglou and G. Tasinato, Nucl. Phys. B677, 405 (2004); J. Garriga and M. Porrati, JHEP, 08, 028 (2004); H. M. Lee, Phys. Lett. B587, 117 (2004); J. W. Chen, M. A. Luty and E. Ponton, JHEP, 09, 012 (2000); C. P. Burgess et al, JHEP, 11, 069 (2004); J. Vinet and J. M. Cline, Phys. Rev. D70, 083514 (2004); Phys. Rev. D71, 064011, (2005). 
[9] T. Gherghetta, M. E. Shaposhnikov, Phys. Rev. Lett. 85,230 (2000); I. Brevik et al, Phys. Rev. D 66, 064016 (2002); K. Ghoroku, M. Yahiro, Phys. Rev. D 66, 124020 (2002).

[10] W.D.Goldberger and M.B.Wise, Phys.Rev.Lett.83,4922 (1999).
[11] Our Eq.(8) is equivalent to Eq.(17) of T. Shiromizu, K. Maeda, M. Sasaki, Phys. Rev. D62 (2000) 024012, in the absence of matter on the brane.

[12] We thank A. Sen for pointing this out to us. 